\title{
The High Time Resolution Spectral Evolution of Gamma-Ray Bursts
}

\author{
David L. Band* and Lyle A. Ford ${ }^{\dagger}$ \\ * CASS, UC San Diego, La Jolla, CA 92093 \\ ${ }^{\dagger}$ University of Wisconsin, Eau Claire, WI 54702
}

\begin{abstract}
Previous studies of the evolution of gamma-ray burst spectra have generally relied on fitting sequences of spectra. These studies usually were confined to bright bursts and often lacked sufficient temporal resolution. We have developed techniques which sacrifice spectral resolution for temporal resolution. First, the crosscorrelations between intensity lightcurves in different energy bands allow for the classification of the spectral evolution of a large burst sample. Second, the energy correlation of pairs of counts places limits on short-duration, narrowband emission.
\end{abstract}

\section{INTRODUCTION}

Since the observed gamma-ray burst spectrum reflects the physical processes within the source's emitting region, spectral variations are an important diagnostic of the nature of this region. Studies showed that the spectrum hardens during intensity spikes, but there is a hard-to-soft trend during these spikes, and the hardness tends to peak at successively lower values from spike to spike (e.g., using SIGNE [1] and BATSE [2] spectra). These studies fitted sequences of spectra and then compared the lightcurves of the intensity and a measure of the spectral hardness derived from these fits (e.g., the energy $E_{p}$ of the peak of $E^{2} N(E) \propto \nu F_{\nu}$ ). Since the spectrum must have sufficient counts for a reliable fit, these studies often lacked the temporal resolution to determine the spectral evolution of the structure which is evident in the intensity lightcurves, and the best temporal resolution was available only for the brightest bursts.

The BATSE spectra accumulated over timescales as short as $0.1 \mathrm{~s}$ are well described by the broadband "GRB" spectral model consisting of two power laws which join smoothly [3]. Yet, early models of a cosmological fireball predicted a narrowband, quasi-black body spectrum when the fireball becomes optically thin $[4,5]$. Fireball models have developed greatly in the past decade, and they no longer predict narrowband spectra. Nonetheless, the question remains whether burst spectra are narrowband on short timescales. 
BATSE provides burst data with a variety of temporal and spectral resolutions; usually there is a tradeoff between the two. Here we emphasize temporal resolution at the expense of spectral resolution. First, we used the auto- and crosscorrelation of lightcurves in different energy bands to categorize the evolution in a large burst sample [6]. Second, we searched for short duration, narrowband emission by correlating the energies of pairs of counts as a function of the arrival time separations [7]. This methodology uses individual counts identified by arrival time and energy; the number of such counts is insufficient for forming a spectrum on millisecond timescales.

\section{AUTO- AND CROSSCORRELATIONS OF ENERGY CHANNELS}

To characterize the spectral evolution of a large sample of bursts we use the autoand crosscorrelation functions (ACF and CCFs, respectively) of burst lightcurves in different energy channels [6]. The BATSE LADs provide discriminator rates in 4 energy bands (Ch. 1: 25-50, Ch. 2: 50-100, Ch. 3: 100-300, and Ch. 4: 300$2000 \mathrm{keV}$ ) on a $64 \mathrm{~ms}$ timescale before, during and after a burst. Most temporal analysis assumes that the time series being analyzed is a sample from a stationary process, which justifies the use of periodic functions in the analysis. However, bursts are transient phenomena; ACFs and CCFs do not assume that the signal is stationary.

The ACFs and CCFs of transient events such as bursts involve some subtleties $[8,6]$. Instead of using a zero-mean time series (i.e., the mean value is subtracted from the lightcurve), we use a background-subtracted time series, which is equivalent to a zero-mean time series of a burst with infinite stretches of background before and after the burst. Assuming the noise is dominated by Poisson statistics, we can correct for this noise by modifying the variance. The resulting ACFs and CCFs are very robust, and give the correct shape even for signal-to-noise ratios of order unity at the peaks of the lightcurve. Only when there is little or no apparent signal, as is sometimes the case for the highest energy channel $(E>300 \mathrm{keV})$, is the CCF so noisy as to be unusable.

We calculate the CCFs of a fiducial energy channel, Ch. 3 (100-300 keV), with each of the 4 energy channels (the CCF of the fiducial channel with itself is that channel's ACF). Through simulations and analytic modeling we have developed diagnostics for different types of spectral evolution. We compare the time lags of the peaks of each curve and their relative values at different lags, as shown by the example in Figure 1.

We calculated the ACFs and CCFs for 209 strong, mostly long bursts [6]. The order of the CCF peaks shows that in general high energy emission precedes low energy emission. As was known previously from comparing the ACFs of the different channels [8], the CCF widths indicate that high energy temporal structure is narrower than low energy structure (i.e., spikes last longer at low energy than at 
high). The relative order of the CCFs at different lags shows there is hard-to-soft evolution within and among spikes in $\sim 80-90 \%$ of the bursts, and there are only a few cases of soft-to-hard spectral evolution. The peaks of the CCFs for the high energy channels typically lead those of the low energy channels by $0.1-0.2 \mathrm{~s}$, and the CCFs of the low energy channels are typically $\sim 25 \%$ broader (where the CCFs have a value of 0.5 ) than those of the high energy channels. Thus this study shows that hard-to-soft spectral evolution is ubiquitous but counterexamples exist.

\section{BROADBAND VS. NARROWBAND EMISSION}

In burst spectra the photons are distributed over a broader energy band than a simple black body spectrum. The question is whether the spectrum is inherently broadband or is composed of short duration narrowband (e.g., black body) events which rapidly sum to a broadband spectrum. Narrowband spectra may indicate thermal processes. BATSE provides full spectra only on timescales of $0.128 \mathrm{~s}$ or
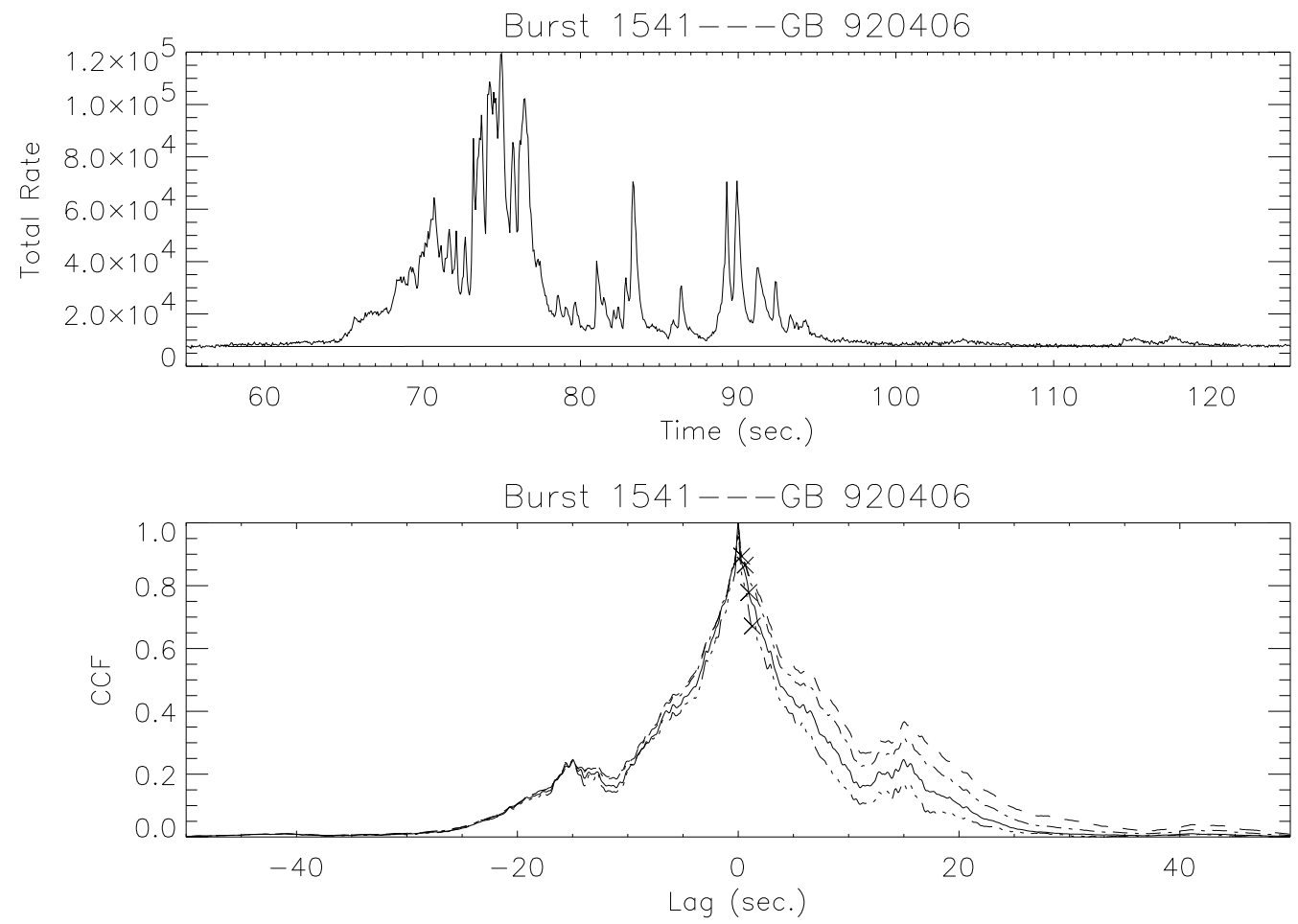

FIGURE 1. Lightcurve (top) and the ACF and CCFs (bottom) of GRB 920406. The solid curve is the ACF of the fiducial energy channel (Ch. 3), the 3 dots-dashed curve is the CCF with a higher energy channel (Ch. 4), and the two remaining curves are the CCFs with lower energy channels (Ch. 1-dashed - and Ch. 2-dot-dashed). The order of the correlation curves indicate the type of spectral evolution. Specifically, for hard-to-soft evolution the order (from top to bottom) of the curves on the positive lag side is: dashed, dot-dashed, solid and 3 dots-dashed. 
longer; spectra can be formed on shorter timescales from the SD time-tagged events (the STTE datatype provides a list of 64,000 counts with their arrival time to $128 \mu \mathrm{s}$ and energy in one of 256 channels), but the photon flux is insufficient to accumulate spectra on timescales of 1-10 ms. Consequently we searched for short duration narrowband events by correlating the energies of pairs of counts [7]. If there is short duration narrowband emission, then the energies of count pairs with a small arrival time separation should be more correlated than counts with a large separation.

Thus we would like the fraction of count pairs whose energies differ by less than a certain fraction of their average energy (i.e., those pairs for which $\left|E_{1}-E_{2}\right|<$ $\left.f\left(E_{1}+E_{2}\right) / 2\right)$. However, since the detectors are imperfect, the counts assigned to a given apparent energy bin could have originated from a broad energy range. With assumptions about the incoming photon spectrum, the detector response can be inverted to provide a distribution $p\left(E \mid E^{\prime}\right)$ of the likely photon energy $E$ given the apparent energy $E^{\prime}$ (the energy reported by the detector). These distributions are used to calculate the likely probability the two counts had energies within a certain fractional separation:

$$
L\left(E_{1}^{\prime}, E_{2}^{\prime}\right)=\int_{0}^{\infty} d E \int_{-f E /(1+f)}^{f E /(1-f)} d(\Delta E) p\left(E \mid E_{1}^{\prime}\right) p\left(E+\Delta E \mid E_{2}^{\prime}\right)
$$

This probability $L\left(E_{1}^{\prime}, E_{2}^{\prime}\right)$ is then averaged over all pairs of counts with a separation $\Delta t$, resulting in a function $A(\Delta t)$.

This function $A(\Delta t)$ should be constant if the spectrum is constant, but $A(\Delta t)$ will increase for decreasing $\Delta t$ if there is short duration narrowband emission, as has been verified by simulations (see the lefthand side of Figure 2). This methodology will identify narrowband emission superimposed on a broadband spectrum. We are quantifying the fraction of narrowband emission on a given timescale which is
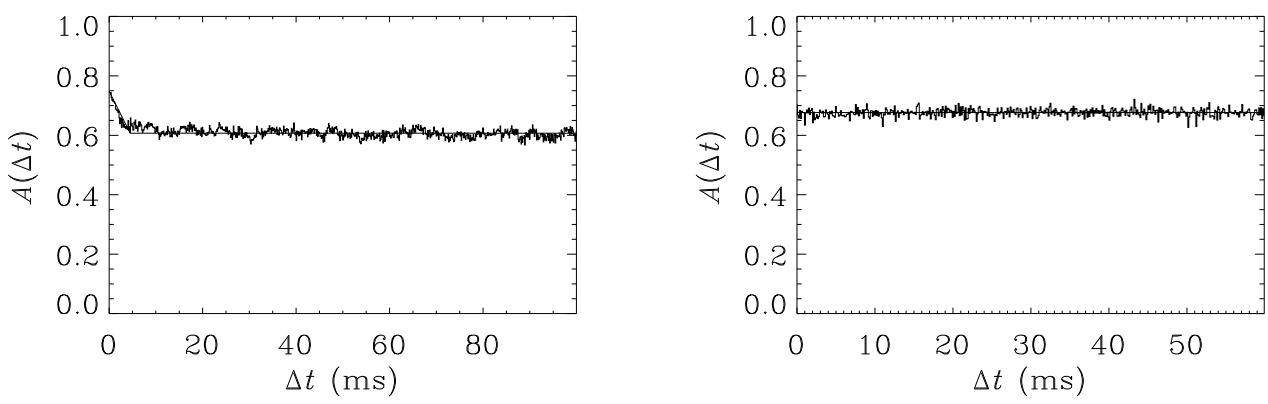

FIGURE 2. The energy separation statistic $A(\Delta \tau)$ as a function of the time separation. On the left is a simulation where the spectrum is formed from black bodies with a distribution of temperatures and durations, while on the right is data from GRB 940717. Note the upturn in $A(\Delta \tau)$ for the simulation with narrowband emission, and the absence of an upturn for the actual data. 
consistent with the data; we anticipate that this fraction will usually be an upper limit.

This methodology has been applied to 20 bright, short duration (less than $2 \mathrm{~s}$ ) bursts. No evidence of short duration narrowband emission was found (see the right-hand side of Figure 2 for an example), with upper limits on the fraction of such emission of order $10 \%$ on timescales down to $1 \mathrm{~ms}$. A complication which needs to be solved is the normal spectral evolution on timescales longer than we are interested.

B. Schaefer (private communication, 1997) is undertaking a similar study using the LAD's Time Tagged Events (TTE) datatype which provides 16,000 counts in 4 energy channels to $2 \mu$ s accuracy.

\section{ACKNOWLEDGMENTS}

The work of the UCSD group is supported by NASA contract NAS8-36081.

\section{REFERENCES}

1. Kargatis, V., et al., Ap. J. 422, 260 (1994).

2. Ford, L. A. et al., Ap. J. 439, 307 (1995).

3. Band, D., et al., Ap. J. 413, 281 (1993).

4. Goodman, J., Ap. J. Lett. 308, L47 (1986).

5. Paczyński, B., Ap. J. Lett. 308, L43 (1986).

6. Band, D. L., Ap. J. 486, 928 (1997).

7. Ford, L. A., Ph.D. Thesis, UC San Diego (1996).

8. Fenimore, E. E., et al., Ap. J. Lett. 448, L101 (1995). 